\title{
Automatic Labeling Inconsistencies Detection And Correction For Sentence Unit Segmentation In Conversational Speech
}

\author{
Sébastien Cuendet ${ }^{1}$, Dilek Hakkani-Tür ${ }^{1}$, and Elizabeth Shriberg ${ }^{1,2}$ \\ ${ }^{1}$ International Computer Science Institute (ICSI) \\ 1947 Center Street \\ Berkeley, CA 94704, USA \\ ${ }^{2}$ Speech Technology and Research Laboratory, SRI International \\ 333 Ravenswood Avenue \\ Menlo Park, CA 94025, USA \\ \{cuendet, dilek, ees\}@icsi.berkeley.edu
}

\begin{abstract}
In conversational speech, irregularities in the speech such as overlaps and disruptions make it difficult to decide what is a sentence. Thus, despite very precise guidelines on how to label conversational speech with dialog acts (DA), labeling inconsistencies are likely to appear. In this work, we present various methods to detect labeling inconsistencies in the ICSI meeting corpus. We show that by automatically detecting and removing the inconsistent examples from the training data, we significantly improve the sentence segmentation accuracy. We then manually analyze 200 of noisy examples detected by the system and observe that only $13 \%$ of them are labeling inconsitencies, while the rest are errors done by the classifier. The errors naturally cluster into 5 main classes for each of which we give hints on how the system can be improved to avoid these mistakes.
\end{abstract}

Key words: automatic relabeling, error correction, boosting, sentence segmentation, noisy data.

\section{Introduction}

Sentence segmentation from speech is part of a process that aims at enriching the unstructured stream of words output by automatic speech recognizers (ASR). The role of sentence segmentation is to find the sentence units in the stream of words output by the ASR. It is of particular importance for speech related applications, as most of the further processing steps, such as parsing, machine translation, information extraction, assume the presence of sentence boundaries $[1,2]$.

Sentence segmentation can be seen as a binary classification problem, in which every word boundary has to be labeled as a sentence boundary or as a non-sentence boundary. In the usual learning task, when provided with data, one has to manually label a consequent amount of them to perform automatic 
learning. In this work, we focus on sentence segmentation for conversational speech from the ICSI meeting corpus, which has been manually labeled with 5 dialog acts: statement, question, backchannel, floor-grabber/holder, incomplete. Backchannels are short phrases such as yeah or uh huh to indicate that the listener is actually following the speaker. Floor grabbers indicate that the person wants to start talking; similarly floor holders indicate that the speaker has not yet finished. Disruptions (also called incompletes) stand for statements that remain uncompleted for some reason. Figure 1 shows an example of a dialog along with dialog acts. For the sentence segmentation, we merge all DAs into one

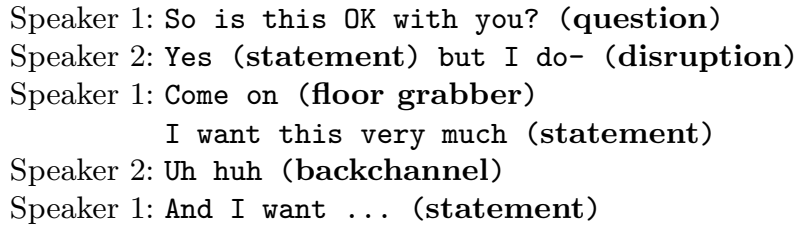

Fig. 1. Example of a dialog along with dialog acts.

class, the "sentence" class, and the goal of the classification is to find the correct locations for the beginning and end of each sentence unit. It is therefore crucial that the DAs have been consistently labeled beforehand. Consistent labeling is however not always guaranteed, since labels are attributed by humans who make mistakes because of the difficulty of DAs labeling in conversational speech. Indeed, conversational speech comprises incomplete and gramatically incorrect sentences which make some candidate boundaries likely to be labeled as sentence boundary as well as non-sentence boundary ${ }^{1}$. Therefore, additionally to the inter-labeler inconsistencies due to a possible different interpretation among the labelers, the complexity of the task leads to inconsistencies. Figure 2 shows a case where the labeler has labeled the word boundary after the word sentence as the end of a statement, but another labeler might as well have not inserted anything and just considered the whole example as one statement. Such inconsistencies in the labeling might confuse the classifier and decrease the sentence segmentation accuracy.

but the phrase is not part of the sentence. and neither is the sentence part of the phrase.

Fig. 2. Example of a dialog along with dialog acts.

In this paper, we study four approaches to automatically detect these ambiguous or wrongly labeled examples. The first approach is based on a committee

${ }^{1}$ More details about the labeling can be found in the guidelines that were given to the labelers in [3]. 
decision, the second one is based on the confidence attributed by the classifier to each instance, and the two last methods use the weights and edges measures of the learning algorithm used, AdaBoost. We show that the sentence segmentation accuracy significantly increases when we remove the noisy examples from the training data, whereas relabeling them does not increase the performance much.

The rest of the paper is structured as follows: in the next section, we describe Boosting, the learning algorithm that we used and review the work done in automatic noise detection. In Section 3, we describe our four approaches to detect noisy examples. The results are presented in Section 4 , and discussed in Section 5 .

\section{The Boosting Algorithm And Related Work}

To perform the binary classification task of sentence segmentation, we use the AdaBoost. $\mathrm{MH}^{2}$ algorithm introduced by Schapire and Singer [4], since it has been shown to be among the best classifiers for the sentence segmentation task [5]. Boosting is an iterative procedure that builds a new weak learner $h_{t}$ at each iteration. Every instance of the training data set is assigned a weight. These weights are initialized uniformly and updated on each iteration so that the algorithm focuses on the instances that were wrongly classified on the previous iteration. At the end of the learning process, the weak learners used on each iteration $t$ are linearly combined to form the classification function:

$$
f(x, l)=\sum_{t=1}^{T} \alpha_{t} h_{t}(x, l)
$$

with $\alpha_{t}$ the weight of the weak learner $h_{t}$ and $T$ the number of iterations of the algorithm, $x$ the example to classify and $l$ the label, with $l \in \mathcal{L}$. The label $l$ with highest score $f(x, l)$ is attributed to $x$. More details on Boosting can be found in $[6]$.

Noisy data has always been a problem in the field of statistical learning. Noise can arise from various sources, such as imprecision or error in the measurement, and labeling errors. Multiple approaches have been tried to identify the noisy instances. A method based on a committee of classifier has been successfully introduced for spoken language understanding in [7]. In [8], E. Eskin presents a technique to detect anomalies and applies it to network intrusion detection. The main idea is to consider two sets of data A and B with corresponding distributions $D_{A}$ and $D_{B}$, one for the regular instances (A) and one for the anomalies (B). At the beginning, all instances belong to A. Each instance is then removed from $\mathrm{A}$ and added to $\mathrm{B}$ and $D_{A}$ and $D_{B}$ are recomputed. The difference between the log likelihood before and after the exclusion of the instance decides if the instance should be moved to B or kept in A. This approach can be used

${ }^{2}$ As is commonly done in the literature, we abusively use the term "Boosting" in this paper to designate the AdaBoost.MH algorithm. 
with any statistical classifier that gives an estimation of the distributions $D_{A}$ and $D_{B}$. Other approaches specific to Boosting have also been tried. In [9], the authors suggest to use the weights over the instances at the end of the training in Boosting to detect the mislabeled instances in part-of-speech tagging. The assumption is that instances that have been wrongly annotated are hard to classify, and thus have a high weight at the end of the training phase. A similar approach is used in [10], where the instances are selected according to their edge value instead of their weight. A detailed presentation of the weight and edge measures is provided in the next section. An interesting approach is presented in [11], where the weights of the attributes as well as the weights of the instances are used to detect the noisy data. The approach is evaluated on endowment insurance records and to our knowledge has not been used on other test sets, which makes it difficult to compare to other methods.

While all the methods introduced above use various measures of Boosting, Oza slightly modifies the Boosting algorithm in order to make it more robust to noise [12]. The main idea in this algorithm is to average the new distribution of the instance weights with the distributions of the previous iterations. Averaging the weights has a regularizing effect which leads to a highest training error bound, but a better generalization error bound. Dealing with the noisy examples is thus done implicitly by the classifier, while all other methods require a post-processing of the noisy instances, such as removing them from the training set or relabeling them.

\section{Approach}

In this section, we present four methods to detect the noisy examples in the training set. Once the noisy examples have been detected, we can either remove them from the training set or automatically relabel them. Relabeling is especially trivial in the case of binary classification, since if an example does not belong to the sentence boundary class, it belongs to the non-sentence boundary class, and inversely.

In the following description, we assume a data set $D$ of training instances, with $|D|=N$. Each example $x_{i}$ in $D$ is represented by a set of features and belongs to a class $y_{i} \in \mathcal{Y}$ that has been assigned by human labelers and to which we refer as the true class. $\mathcal{Y}=\{s, n\}$ is the set of possible classes with $s$ the class of examples which are sentence boundaries and $n$ the class of examples that are non-sentence boundaries. The Boosting algorithm described in the previous section is used to output a probability $p\left(s \mid x_{i}\right)$ for each example $x_{i}$ to belong to the class $s$. If the $p\left(s \mid x_{i}\right)$ is larger or equal to a threshold $T, x_{i}$ is attributed the class $s$, i.e. declared as a sentence boundary, otherwise it gets class $n$, i.e. declared as a non-sentence boundary.

\subsection{Committee-Based Method}

The training set $D$ is split into $k$ mutually exclusive data sets $d_{j}$ of size $N / k$ each. A classifier $c_{j}$ is trained on each of the reduced data sets $d_{j}$. The $k$ classifiers 
$c_{j}$ are then used to evaluate each example in $D$. Therefore, for each instance of the original data set $D$, we now have $k$ votes. An example $x_{i}$ is defined as noisy when all $k$ classifiers $c_{j}$ agree on a class $y_{i}^{\prime}$, and $y_{i}^{\prime}$ is different from the true class $y_{i}$.

We describe 3 variants of this method. One of them is to exclude an example $x_{i}$ if $k^{\prime}$ classifiers agree on a class $y_{i}^{\prime} \neq y_{i}$, where $k^{\prime}<k$. This is a less strong excluding condition and is thus more likely to remove non-noisy examples. Another variant is to remove only the examples whose true class is $n$ whereas the $k$ classifiers have agreed on class $s$ (false positives). The motivation behind this variant is that labelers are more likely to forget to label an instance as a sentence boundary, while it truly is a sentence boundary, than to add sentence boundaries where there is no reason to have sentence boundaries. The last variant is to use a variable threshold $T$. Optimizing the threshold for each of the $k$ classifier would however be computationally too expensive and we therefore only use $T \in\{0.3,0.5\}$.

\subsection{Confidence-Based Method}

The complete training set $D$ is first used to train a classification model $M_{1}$. The model $M_{1}$ is then used to estimate the class of each example $x_{i}$ in $D$. The noisy examples are those that have a true class $y_{i}$ but are assigned a class $y_{i}^{\prime}$ by the classification model $M_{1}$, where $y_{i} \neq y_{i}^{\prime}$ and the probability $p\left(y_{i}^{\prime} \mid x_{i}\right)$ assigned to the class $y_{i}^{\prime}$ for example $x_{i}$ is larger than a threshold $Z$ optimized on the held-out set. In Section 4, we present 3 variants of the experiment: one where all the detected noisy examples are excluded, and two where the false negatives (resp. false positives) are relabeled. Note that the confidence-based method is a special case of the committee-based method, where $k=1$ and the true class in the detecting phase is determined with an optimized threshold.

\subsection{Boosting Weights Method}

This method is based on the observations done in [9] and uses the weights attributed by Boosting to each training instance. We use a simplified version (since sentence segmentation has only two classes) of the original weight update function described in [4]:

$$
\begin{gathered}
W_{t+1}(i)=\frac{W_{t}(i) \exp \left(-h_{t}\left(x_{i}\right) \cdot Y[i]\right)}{Z_{t}} \\
Z_{t}=\sum_{i=1}^{N} W_{t}(i) \exp \left(-h_{t}\left(x_{i}\right) \cdot Y[i]\right)
\end{gathered}
$$

where $W_{t}(i)$ is the weight of instance $x_{i}$ at iteration $t$, and

$$
Y[i]=\left\{\begin{array}{l}
+1, \text { if } y_{i}=s \\
-1, \text { if } y_{i}=n .
\end{array}\right.
$$


Thus, if the current rule $h_{t}$ classifies the example $x_{i}$ incorrectly, the next weight $W_{t+1}(i)$ will increase, otherwise it will decrease. To decide which examples are noisy, we sort all examples according to their weight at the end of the training and declare the top $X$ examples as being noisy.

The parameters are the number $X$ of excluded examples, as well as the number of iterations used to train the classifier. If we train for too many iterations, we take the risk of having the examples that are noisy having their weights decreased because the final rules created by Boosting after several iteration eventually classify them correctly. On the other hand, if we train for too few iterations, the weights of the noisy examples might not have had the chance to increase enough compared to those of the regular examples.

\subsection{Boosting Edges Method}

The definition of edges has first been introduced by Breiman [13], and used to detect noisy data in [10]. The edge value edge $i_{i}$ of an instance $x_{i}$ at iteration $t$ is the total weight assigned to $x_{i}$ by all $h_{t}$ that misclassified $x_{i}$ up to iteration $t$ :

$$
\operatorname{edge}_{i}=\sum_{t=1}^{T} h_{t}\left(x_{i}\right) I_{t}\left(x_{i}\right)
$$

$I_{t}\left(x_{i}\right)$ is the following indicator function, where $\left[h_{m}\left(x_{i}\right)\right]$ is the class assigned by $h_{m}$ to $x_{i}$ :

$$
I_{m}\left(x_{i}\right)=\left\{\begin{array}{l}
0, \text { if }\left[h_{m}\left(x_{i}\right)\right]=y_{i} \\
1, \text { if }\left[h_{m}\left(x_{i}\right)\right] \neq y_{i}
\end{array}\right.
$$

Note that the edge values are always positive since the chosen class is by definition the one that has a positive weight in a two-class Boosting problem.

In [10], Wheway suggests to declare as noisy the $5 \%$ instances with highest edge value after 10-20 iterations. She however does not evaluate her suggestion and although we think this approach is reasonable, we will experimentally show that the percentage of instances declared as noisy, as well as the number of iterations after which the edge values are computed, are both parameters that have to be optimized

\section{Experiments and Results}

Data Sentence segmentation is performed on conversational speech, which comes from the ICSI meeting corpus (MRDA) [14]. This corpus contains 73 meetings which are grouped in three main types (according to the speakers, the conversations type, etc.). We use the same split of training, test and held-out set as specified in [15], i.e. 51 meetings for the training set, 11 meetings for the test set and 11 meetings for the held-out set. More details about the data are shown in Table 1. We use the manual transcriptions of the meetings and feed the classifier with both lexical and prosodic features, for a total of 34 features. The prosodic features are various measures of the pitch, energy and pause duration across the boundary of interest. The lexical features are unigrams, bigrams, and trigrams 


\begin{tabular}{|l|c|}
\hline Training set size (words) & 538,956 \\
Test set size & 101,510 \\
Held-out set size & 110,851 \\
Vocabulary size & 11,034 \\
Average utterance length & 6.54 \\
\hline
\end{tabular}

Table 1. Data characteristics of the MRDA corpus. Sizes and sets are given in number of words.

formed with the words surrounding the word boundary of interest. More details on the features can be found in [5].

Metrics To measure the performance of the sentence segmentation, we use the F-measure and the NIST-SU error. The F-measure is the harmonic mean of the recall and precision measures of the sentence boundaries hypothesized by the classifier to the ones assigned by human labelers. The NIST-SU error rate is the ratio of the number of wrong hypotheses made by the classifier to the number of reference sentence boundaries. So if no boundaries are marked by sentence segmentation, it is $100 \%$, but it can exceed $100 \%$; the maximum error rate is the ratio of number of words to the number of correct boundaries.

\subsection{Results}

We now report the results for each of the methods introduced in Section 3. The baseline was obtained by training the classifier on the entire training set and evaluating the classification accuracy on the test set, with parameters optimized on the held-out set. The baseling settings yielded a $81.7 \%$ F-Measure and a $35.6 \%$ NIST-SU error rate. For each of the methods described above, we present results obtained by optimizing the parameters on the held-out set.

For the committee-based method, we tried values 8,9 and 10 for $k$, values 0.3 and 0.5 for the Boosting threshold, and for each of the settings, we tried to exclude only the noisy examples that the labelers labeled with class $n$. The results of the 2 best settings on the held-out set for each value of $k$ are shown in Table 2.

For the high confidence disagreement method, the optimal value for the threshold $Z$ on the held-out set was 0.6. Table 3 shows the results when we excluded noisy examples or relabeled a subset of them.

For the weights and the edges experiment, we trained Boosting for $M$ iterations, with $M \in \mathcal{M}=\{10,20,50,100,200,300,400,500,1000\}$ iterations. For each $M \in \mathcal{M}$ iteration, we removed the $X$ examples with the top weight (resp. edge) score, with $X \in[1000,2000, \ldots, 10000]$, and report the results in Tables 4 and 5 for the number of excluded examples $X$ that yielded the best result on the held-out set. Note that when several examples had the same score and they were at the border of the $X$ top examples, we excluded all examples that had the exact same value as the $X^{t h}$ example. 


\begin{tabular}{|c|c|c|c|c|}
\hline$k$ & Ths. & \# Noisy (\% of total) & F-Meas. & NIST err. \\
\hline \multicolumn{2}{|c|}{ Baseline } & 0 & 81.71 & 35.59 \\
\hline 10 & 0.5 & 10,786 & $82.10(2.0)$ & 35.07 \\
\hline 10 & 0.3 & 9,363 & $82.11(1.7)$ & 34.99 \\
\hline 9 & 0.5 & 21,572 & $81.66(4.0)$ & 35.01 \\
\hline 9 & 0.3 & 18,726 & $82.09(3.5)$ & 34.90 \\
\hline 8 & 0.5 & 22,465 & $81.96(4.2)$ & 34.63 \\
\hline 8 & 0.3 & 22,016 & $82.35(4.1)$ & 34.32 \\
\hline
\end{tabular}

Table 2. Results for the committee-based method. The first column shows the number of votes required to tag an example as noisy, the second shows the threshold that distinguishes between the two classes $n$ and $s$; the 3 last columns show the result for the setting of the 3 first columns: number of examples excluded and accuracy according to the F-Measure and the NIST error.

\begin{tabular}{|c|c|c|c|}
\hline Processing & \# Noisy (\% of total) & F-Meas. & NIST err. \\
\hline Baseline & 0 & 81.71 & 35.59 \\
\hline Exclude all noisy & $22,951(4.3)$ & 82.00 & 34.6 \\
\hline Relabel false positive & - & 82.00 & 34.7 \\
\hline Relabel false negative & - & 82.10 & 34.7 \\
\hline
\end{tabular}

Table 3. Results for the confidence-based method. The first row shows the standard case described in the text, while for the results in rows 2 and 3, only the examples with true class $s$ (resp. $n$ ) were kept, while examples from the other class were relabeled.

All presented methods outperformed the baseline with optimized parameters. The overall improvement can look small, but an F-Measure above $81.94 \%$ and a NIST error under $35.30 \%$ are both statistically significant improvements according to a Z-test with $95 \%$ confidence range. The overall best performance was obtained by the committee-based method with $k=8$ and improved the baseline of $0.7 \%$ absolute for the F-Measure and $1.3 \%$ absolute for the NIST error. In some settings, the F-Measure was lower than for the baseline, as opposed to the NIST error which was always better than the baseline, which means that in any of these settings, the number of wrong word boundary predictions done by the new classifier was lower than in the baseline.

The optimal parameters used for the edges method was different than those in [10], where the author suggests to stop the training after 10-20 iterations and to exclude the top $5 \%$ examples. Our optimal solutions used 100 iterations and excluded less than $2 \%$ of the examples.

Removing vs. Relabeling Examples The methods presented above determine which examples were considered as noisy but not how to handle them. Once we have detected noisy examples, we can either remove them from the training set, or we can try to automatically relabel them. Since the sentence segmentation problem is a binary classification problem, relabeling is straightforward: noisy 


\begin{tabular}{|c|c|c|c|}
\hline Iterations & \# Noisy (\% of total) & F-Meas. & NIST err. \\
\hline Baseline & 0 & 81.71 & 35.59 \\
\hline 10 & 1,000 & $81.34(0.2)$ & 35.48 \\
\hline 20 & 1,000 & $81.82(0.2)$ & 35.45 \\
\hline 50 & 1,000 & $81.66(0.2)$ & 35.66 \\
\hline 100 & 6,000 & $81.76(1.1)$ & 35.27 \\
\hline 200 & 6,000 & $81.94(1.1)$ & 35.24 \\
\hline 300 & 7,000 & $81.74(1.3)$ & 35.15 \\
\hline 400 & 8,000 & $81.95(1.5)$ & 35.02 \\
\hline 500 & 8,000 & $81.97(1.5)$ & 34.87 \\
\hline 1000 & 10,000 & $81.83(1.9)$ & 34.87 \\
\hline
\end{tabular}

Table 4. Results for the weights methods. The first column shows the number of iterations after which the weight values are measured, the second column indicates the number of examples tagged as noisy and the last two columns report the sentence segmentation accuracy according to the F-Measure and the NIST error.

examples originally labeled with class $s$ are changed to class $n$ and vice versa. However, in all of our experiments, we observed that although better than the baseline, the performance obtained after relabeling the noisy examples was lower or equal to the one obtained by simply excluding them. One explanation for this is that the noisy relabeled examples do not bring much new knowledge to the classifier, while examples that were correctly labeled and detected as noisy add noise to the data when they are relabeled.

\section{Discussion}

In the previous section, we have shown that the sentence segmentation accuracy improves when we exclude the noisy examples. While this is already a valuable result, we believe there is more knowledge to extract from the noisy examples. In the rest of this section, we examine the noisy examples for the committeebased method with $k=10$ and the exclusion of examples from the two classes. In this setting, the system detected 10,786 noisy examples; $23 \%$ of them are instances where the system introduced an additional sentence boundary, while the remaining $77 \%$ are sentence boundaries that the system missed.

Among all the examples whose true class is a sentence boundary, we observe that only $0.32 \%$ of the backchannels are noisy, $15.87 \%$ of the incompletes, $10.66 \%$ of the statements, $12.99 \%$ of the floor-grabbers/holders and $11.95 \%$ for the questions. This confirms the intuition that disruptions are the most difficult cases to label.

One possible source of errors in this work could be human mistakes in assigning original dialog act boundaries. To explore this possibility, and further understand errors made by the system, a researcher familiar with the original dialog act annotation project hand-analyzed 200 randomly drawn errors using 


\begin{tabular}{|c|c|c|c|}
\hline Iterations & \# Noisy (\% of total) & F-Meas. & NIST err. \\
\hline Baseline & 0 & 81.71 & 35.59 \\
\hline 10 & $2,000(0.4)$ & 81.52 & 35.31 \\
\hline 20 & $1,000(0.2)$ & 81.84 & 35.31 \\
\hline 50 & $3,000(0.6)$ & 82.00 & 34.95 \\
\hline 100 & $4,000(0.7)$ & 82.18 & 34.45 \\
\hline 200 & $5,000(0.9)$ & 82.03 & 34.68 \\
\hline 300 & $6,000(1.1)$ & 81.94 & 35.06 \\
\hline 400 & $8,000(1.5)$ & 81.67 & 35.16 \\
\hline 500 & $10,000(1.9)$ & 81.83 & 35.04 \\
\hline 1000 & $6,000(1.1)$ & 82.00 & 34.72 \\
\hline
\end{tabular}

Table 5. Results for the edges methods. The first column shows the number of iterations after which the edge values are measured, the second column indicates the number of examples tagged as noisy and the last two columns report the sentence segmentation accuracy according to the F-Measure and the NIST error.

transcripts only, but with information about reference human punctuation labels, including disfluency markers and markers for incomplete sentences. Speech from other talkers was also interspersed in the transcripts, and the length of pauses was supplied. Of the original 200 examples, $10 \%$ were found difficult to understand from transcripts alone; the analysis thus refers to the remaining 178 samples. Of this set, only $13 \%$ were errors in the original human boundary labels, with a nearly even split between missed boundaries and false alarms. Because the analysis looks only at errors to begin with, this rate of human labeling error is tolerable (although to estimate it properly would also require determining the rate of felicitous correct machine decisions due to erroneous human labels). The remaining of the 178 cases were deemed to have correct human boundary labels.

The analysis becomes more interesting as we look at the remaining errors, all attributable to the system. Percentages are given as the percentage of the 178 original cases referred to above. Over half $(54 \%)$ of the remaining errors fell into one of five groups. The first group, at 15\%, had either a false start or incomplete sentence preceding the boundary of interest. In a two-way classification of boundaries there is no good way to group such cases, since to the left of the disruption they reflect no boundary, but to the right of the disruption they begin a new sentence and thereby suggest a boundary. To handle such cases explicitly, one would need to train specific models for this third boundary type. The second group, at $14 \%$, comprises boundaries directly following filled pauses or discourse markers. Considering floor-grabbers/holders as full sentence boundaries, as explained in Section 1, is certainly the cause of this second class of errors. Since these boundaries are not per se sentence boundaries, one way of dealing with them would be to simply consider them as non-sentence boundaries or to treat them as a separate class. The third class of errors, which really should not be counted as errors at all, are ambiguous examples in which a human would have trouble assigning boundaries. An example of such a case is shown 
in Figure 3, where the word boundary after the word document was labeled as a statement, but considering it as a non-sentence boundary would clearly be correct too. Fourth, boundaries after questions accounted for $9 \%$ of errors. It

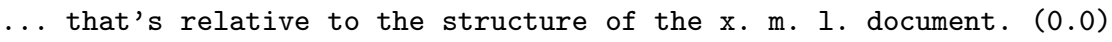

Fig. 3. Example tagged as noisy by the committee-based method. The parenthesis indicate the length of the pause between the 2 words document and not.

is likely that the model suffers here both because question prosody often leaves pitch high, unlike the majority of boundaries occurring for statements that show final falls. The language model may also have trouble with questions, which can end in verbs or other syntactic classes that are unusual for sentence ends in statements. Finally, about $5 \%$ of cases occurred at subsentential locations that in text should contain a colon or semicolon. Such errors can be viewed similarly to the errors made for disfluency boundaries: the subsentential boundaries really belong somewhere in between the no-boundary and boundary classes. The remaining machine errors (at $33 \%$ of the 178 samples) had no obvious cause. Within this set, missed boundaries were three times as likely as false alarms. We can balance this ratio by setting the threshold to less than 0.5 and thus globally detect more sentence boundaries, and this 3 to 1 ratio is thus not a general rule and depends on the optimal threshold.

\section{Conclusion}

We presented four automatic methods to detect labeling error for automatic sentence segmentation. Although tested only on the ICSI meeting corpus, the methods can be applied to other conversational speech data, such as broadcast conversation and telephone conversations. We showed that the sentence segmentation accuracy improved when the noisy examples were first excluded from the training set, with either of the four methods. Relabeling the noisy instead of excluding them did not further improve the performance. We analyze 200 noisy examples: $13 \%$ were found to be labeling errors, $54 \%$ were errors done by the system that we could explain and that could be clustered into 5 main classes, and the rest of them were errors done by the system for which there was no clear explanation.

Further work will consist of using the knowledge extracted from the noisy examples to improve the sentence segmentation accuracy. Significant improvement can especially be obtained by focusing particularly on incompletes and questions detection.

Acknowledgments. We want to thank Mathew Magimai Doss for many helpful discussions. This work was partly supported by the Swiss National Science 
Foundation through the research network IM2 and Defense Advanced Research Projects Agency (DARPA) GALE (HR0011-06-C-0023). Any opinions, findings, and conclusions or recommendations expressed in this material are those of the authors and do not necessarily reflect the views of the funders.

\section{References}

1. Mrozinski, J., Whittaker, E.W.D., Chatain, P., Furui, S.: Automatic sentence segmentation of speech for automatic summarization. In: Proc. ICASSP, Philadelphia, PA (2005)

2. Makhoul, J., Baron, A., Bulyko, I., Nguyen, L., Ramshaw, L., Stallard, D., Schwartz, R., Xiang, B.: The effects of speech recognition and punctuation on information extraction performance. In: In Proc. of Interspeech, Lisbon (2005)

3. Shriberg, E., Dhillon, R., Bhagat, S., Ang, J., , Carvey, H.: The ICSI meeting recorder dialog act (MRDA) corpus. In: Proc. SigDial Workshop, Boston, MA (2004)

4. Schapire, R.E., Singer, Y.: BoosTexter: A boosting-based system for text categorization. Machine Learning 39(2/3) (2000) 135-168

5. Zimmermann, M., Hakkani-Tür, D., Fung, J., Mirghafori, N., Shriberg, E., Liu, Y.: The ICSI+ multi-lingual sentence segmentation system. In: Proc. ICSLP, Pittsburgh, PA (2006)

6. Schapire, R.: The boosting approach to machine learning: An overview. In: In MSRI Workshop on Nonlinear Estimation and Classification, Berkeley, CA (2001)

7. Tur, G., Rahim, M., Hakkani-Tür, D.: Active labeling for spoken language understanding. In: Proceedings of EUROSPEECH, Geneva, Switzerland (2003)

8. Eskin, E.: Anomaly detection over noisy data using learned probability distributions. In: Proc. 17th International Conf. on Machine Learning, Morgan Kaufmann, San Francisco, CA (2000) 255-262

9. Abney, S., Schapire, R., Singer, Y.: Boosting applied to tagging and pp attachment. In: Proceedings of the Joint SIGDAT Conference on Empirical Methods in Natural Language Processing and Very Large Corpora, 1999. (1999)

10. Wheway, V.: Using boosting to detect noisy data. In: Revised Papers from the PRICAI 2000 Workshop Reader, Four Workshops held at PRICAI 2000 on Advances in Artificial Intelligence, London, UK, Springer-Verlag (2001) 123-132

11. Liu, X.D., Shi, C.Y., Gu, X.D.: A boosting method to detect noisy data. In: Proc. of the Fourth International Conference on Machine Learning and Cybernetics, Guangzhou, China (August 2005)

12. Oza, N.C.: Aveboost2: Boosting for noisy data. In: Fifth International Workshop on Multiple Classifier Systems, Cagliari, Italy, Springer-Verlag (June 2004) 31-40

13. Breiman, L.: Arcing the edge. Technical report, Statistics Department, UC Berkeley (1997)

14. Janin, A., Ang, J., Bhagat, S., Dhillon, R., Edwards, J., Macias-Guarasa, J., Morgan, N., Peskin, B., Shriberg, E., Stolcke, A., Wooters, C., Wrede, B.: The ICSI meeting project: Resources and research. In: Proceedings of ICASSP, Montreal (2004)

15. Ang, J., Liu, Y., Shriberg, E.: Automatic dialog act segmentation and classification in multiparty meetings. In: Proc. ICASSP, Philadelphia, PA (2005) 